\section{Case Reports in Neurology}

\title{
Infliximab-Associated Chronic Inflammatory Central Nervous System Disease and Peroneal Nerve Injury in a Psoriatic Patient Refractory to Treatment: Case Report with 10-Year Follow-Up
}

\author{
Sandra C. Signore ${ }^{a}$ Birka Brauns ${ }^{b} \quad$ Gunther Schütze $^{c}$ \\ Christoph P. Dohm ${ }^{d}$ Mathias Bähr ${ }^{a} \quad$ Rotraut Mössner $^{\mathrm{e}}$ Pawel Kermer $^{\dagger}$ \\ aDepartment of Neurology, University Hospital Göttingen, Göttingen, Germany; \\ ${ }^{b}$ Department of Dermatology, University Hospital Rostock, Rostock, Germany; ' Radiology \\ Practice, Am Bethanien Krankenhaus, Frankfurt am Main, Germany; ${ }^{d}$ Department of \\ Neurorehabilitation, Krankenhaus St. Elisabeth, Damme, Germany; ${ }^{e}$ Department of \\ Dermatology, University Hospital Göttingen, Göttingen, Germany; ${ }^{\mathrm{f}}$ Department of \\ Neurology, Nordwest-Krankenhaus Sanderbusch, Sande, Germany
}

\section{Keywords}

Infliximab · Chronic inflammatory central nervous system disease · Peroneal nerve injury . Treatment-refractory psoriasis

\begin{abstract}
The tumor necrosis factor- $\alpha$ (TNF- $\alpha$ ) antagonists infliximab, adalimumab, and etanercept have been approved for the treatment of chronic inflammatory diseases such as rheumatoid arthritis, ankylosing spondylitis, psoriasis, and psoriatic arthritis. Manifestations of demyelinating disease have been reported for patients receiving TNF- $\alpha$ antagonists. We describe a rare manifestation of a chronic inflammatory process affecting both the central and peripheral nervous system in a patient who received infliximab for the treatment of psoriasis and
\end{abstract}




\section{Case Reports in Neurology}

psoriatic arthritis. Infliximab therapy was discontinued and symptoms improved under highdose intravenous glucocorticoid pulse therapy.

(C) 2018 The Author(s)

Published by S. Karger AG, Basel

\section{Case Report}

A 45-year-old male patient with chronic plaque psoriasis since the age of 26 developed joint symptoms with pain and swelling 10 years ago. Arterial hypertension, which was well controlled by the antihypertensive drug amlodipine, was the only known comorbidity. Serological investigations including serology for Lyme borreliosis, antinuclear antibodies, antibodies for anti-dsDNA, rheumatoid factor, and uric acid were negative or within normal limits, and the diagnosis of psoriatic arthritis was established. After therapies with oral methotrexate and cyclosporine had proved ineffective in controlling joint and skin symptoms, a therapy with tumor necrosis factor- $\alpha$ (TNF- $\alpha$ ) antagonists was initiated. Both subcutaneous etanercept at a dose of $2 \times 25 \mathrm{mg} /$ week over 6 months and subcutaneous adalimumab at a dose of $40 \mathrm{mg}$ every other week over 4 months were discontinued because of insufficient improvement of skin and joint symptoms. In June 2008, intravenous therapy with infliximab ( $5 \mathrm{mg} / \mathrm{kg}$ every 8 weeks) was initiated. Two months into therapy joint and skin symptoms were greatly improved, with a reduction of the Psoriasis Area and Severity Index from 23.4 prior to infliximab therapy (Fig. 1a) to 3.9, and the therapeutic effect could be maintained throughout the following months of therapy (Fig. 1b).

In January 2009, 1 month after the fourth infusion, the patient noticed weakness of the left arm and right leg with hypesthesia in the absence of other accompanying symptoms such as fever or bladder or bowel incontinence. The patient's family history was negative for multiple sclerosis (MS) and other acute or chronic inflammatory central nervous system (CNS) or peripheral nervous system (PNS) diseases.

Neurological examination revealed a paresis of $4 / 5$ strength at the proximal right leg and $3 / 5$ at the distal right leg. A central paresis of $4 / 5$ at the left arm was also documented. The left arm and right leg showed hypesthesia which could not be described by dermatomes or peripheral nerve territories. Tendon reflexes were decreased on the upper and lower extremities.

Electrophysiological examination revealed a proximal and distal axonal neuropathy of the peroneal nerves (right > left) without proximal conduction block at the neck of the fibula. F-waves were not detectable. Additional electromyography showed chronic nerve damage in the right anterior tibial muscle without acute denervation revealing additional peripheral nerve damage.

A complete blood cell count, liver enzymes, electrolytes, thyroidal gland hormones, and urine values were all within normal limits. MRI scans in February 2009 revealed a lesion in the left peritrigonal region (Fig. 2a, b, arrows) which showed no contrast enhancement, but due to its localization and shape, an inflammatory process was most likely. Lumbar puncture showed a slightly elevated cell count of 5 cells $/ \mathrm{mm}^{3}$ (normal range: $0-4$ cells $/ \mathrm{mm}^{3}$ ) and a mildly increased total protein level of $531 \mathrm{mg} / \mathrm{L}$ (normal range: $150-450 \mathrm{mg} / \mathrm{L}$ ). An elevation of immunoglobulin $\mathrm{G}$ with presence of oligoclonal bands supported the findings of an inflammatory component, which was not explainable due to other causes since there was no acute infection and due to the negative patient history. Clinical examination and neurological workup suggested the diagnosis of an inflammatory process affecting both the CNS and the PNS, whereas the chronic inflammatory CNS disease did not fulfil the diagnostic criteria for MS. Infliximab therapy was discontinued and the patient treated with high-dose intravenous 
methylprednisolone ( $1 \mathrm{~g}$ /day i.v. over 3 days), resulting in a marked improvement of pareses and hypesthesia of the upper and lower extremities.

At discharge, the patient denied any hypesthesia and pareses were barely detectable. In line with lacking exacerbation of neurological symptoms upon control examination 4 weeks later, MRI scans showed a regression of the left peritrigonal lesion, underlining the primary suspicion of an inflammatory lesion. Nine months later, hypesthesia of the left arm was still absent, while paresis of the right foot and hypesthesia of the right anterior lower leg had recurred. The patient received a second cycle of high-dose intravenous methylprednisolone pulse therapy ( $1 \mathrm{~g} /$ day i.v. for 3 days) which resulted in a rapid improvement of hypesthesia and paresis, but not in a complete disappearance of symptoms. At further follow-up examinations, a paresis of the distal right leg of $4 / 5$ strength and hypesthesia in an area around the ankle of the right foot remained.

Currently, after 10 years, the patient is in stable neurological condition. Electrophysiological results such as neuropathy of the peroneal nerves deteriorated, and the patient also showed pathological visual evoked potentials on both sides, further confirming the chronic inflammatory CNS disease, but not fulfilling the criteria for MS yet. At the moment he is getting ustekinumab regularly as dermatologic treatment without suffering severe side effects.

\section{Discussion}

Manifestations of demyelinating disorders have been reported in association with TNF- $\alpha$ antagonists [1, 2], especially for adalimumab [3], etanercept [4], and infliximab [5]. Patients typically suffer from a variety of symptoms such as paresis, paresthesia, optic neuritis, and gait disturbances, with usually a relatively good outcome after discontinuation of anti-TNF drugs, sometimes in combination with usual treatments for demyelinating neuropathies [1-6]. According to the literature, TNF antagonist therapies can be associated with Guillain-Barré and Miller Fischer syndrome [7]. Furthermore, there are cases described in whom TNF- $\alpha$ antagonists induced MS and transverse myelitis [1,2] and vasculitis $[2,8]$. With respect to several publications describing demyelinating diseases during anti-TNF- $\alpha$ treatment, there are also cases with peripheral mononeuropathy [9], multifocal motor neuropathy [10], and polyradiculoneuropathy [11] described.

TNF- $\alpha$ is a proinflammatory and immunoregulatory cytokine which is believed to be involved in the pathogenesis of several CNS disorders such as MS, myasthenia gravis, and amyotrophic lateral sclerosis $[2,11,12]$. As TNF is able to induce the production of other cytokines and adhesion molecules for leukocyte migration [13], it can also have neurotoxic effects by the production of reactive oxygen species. On the other hand, neuroprotective effects of TNF- $\alpha$ have been described $[14,15]$.

After discontinuing the reported patient's infliximab therapy and initiating high-dose methylprednisolone pulse therapy, the pareses improved. Based on clinical examination, lumbar puncture, and MRI studies, the patient suffers from chronic inflammatory CNS disease, but does not fulfil the diagnostic criteria for MS. The differential diagnosis of a vascular CNS lesion caused by hypertonia was unlikely due to localization, shape, and reduction after high-dose infusion with methylprednisolone. Due to the chronological association of the manifestation of the neurological symptoms with infliximab therapy, it is likely that this drug triggered or caused their manifestation, particularly as the patient's family history was negative for MS and other acute or chronic inflammatory CNS diseases. Still, it cannot be excluded that the patient already had a latent chronic inflammatory CNS disease as neither MRI nor 
lumbar puncture were performed prior to initiation of infliximab therapy. This view is supported by the fact that electrophysiological measurements proved some progression over the follow-up time of almost 10 years. In addition, it could be possible that not only infliximab, but also the other two TNF antagonists that the patient had taken previously contributed to the manifestation of neurological symptoms or that their side effects were enhanced.

In summary, this case shows a rare manifestation of a combined chronic inflammatory CNS and PNS disease during infliximab therapy which, to our knowledge, has not been reported yet. It shows the importance of follow-up examinations, including neurological status, for patients with an anti-TNF- $\alpha$ therapy for early detection of inflammatory diseases in the nervous system. In these cases, discontinuation of the anti-TNF- $\alpha$ therapy and the initiation of a therapy for chronic inflammatory disease is recommended.

\section{Statement of Ethics}

All authors hereby state that the patient gave his informed consent for publication of this case report and that all applicable subject protection guidelines and regulations were followed in the conduct of this research.

\section{Disclosure Statement}

There are no conflicts of interest.

\section{References}

1 Zhu TH, Nakamura M, Abrouk M, Farahnik B, Koo J, Bhutani T: Demyelinating disorders secondary to TNF-inhibitor therapy for the treatment of psoriasis: a review. J Dermatolog Treat 2016;27:406-413.

-2 Theibich A, Dreyer L, Magyari M, Locht H: Demyelinizing neurological disease after treatment with tumor necrosis factor alpha-inhibiting agents in a rheumatological outpatient clinic: description of six cases. Clin Rheumatol 2014;33:719-723.

-3 Berthelot CM, George SJ, Hsu S: Distal lower paresthesia and foot drop developing during adalimumab therapy. J Am Acad Dermatol 2005;53:260-262.

-4 Sukal SA, Nadiminti L, Granstein RD: Etanercept and demyelinating disease in a patient with psoriasis. J Am Acad Dermatol 2006;54:160-164.

-5 Lozeron P, Denier C, Lacroix C, Adams D: Long-term course of demyelinating neuropathies occurring during tumor necrosis factor-alpha-blocker therapy. Arch Neurol 2009;66:490-497.

-6 Felekis T, Katsanos K, Christodoulou D, Asproudis I, Tsianos EV: Reversible bilateral optic neuritis after infliximab discontinuation in a patient with Crohn's disease. J Crohns Colitis 2009;3:212-214.

-7 Shin IS, Baer AN, Kwon HJ, Papadopoulos EJ, Siegel JN: Guillain-Barré and Miller Fisher syndromes occurring with tumor necrosis factor alpha antagonist therapy. Arthritis Rheum 2006;54:1429-1434.

-8 Jarrett SJ, Cunnane G, Conaghan PG, Bingham SJ, Buch MH, Quinn MA, Emery P: Anti-tumor necrosis factor-alpha therapy-induced vasculitis: case series. J Rheumatol 2003;30:2287-2291.

-9 Hanaoka BY, Libecco J, Rensel M, Hajj-Ali RA: Peripheral mononeuropathy with etanercept use: case report. J Rheumatol 2008;35:182.

10 Kelly S, Connolly S, McCarthy A, Hutchinson M, Murphy S, Turbridy N: PONM12. Multifocal motor neuropathy due to infliximab. J Neurol Neurosurg Psychiatry 2010;81:63.

11 Solomon AJ, Spain RI, Kruer MC, Bourdette D: Inflammatory neurological disease in patients treated with tumor necrosis factor alpha inhibitors. Mult Scler 2011;17:1472-1487.

12 Ghezzi P, Mennini T: Tumor necrosis factor and motoneuronal degeneration: an open problem. Neuroimmunomodulation 2001;9:178-182. 


\section{Case Reports in Neurology}

\begin{tabular}{l|l}
\hline Case Rep Neurol 2018;10:12-17 \\
\hline DOI: $10.1159 / 000485499$ & $\begin{array}{l}\text { (c) 2018 The Author(s). Published by S. Karger AG, Basel } \\
\text { www.karger.com/crn }\end{array}$ \\
\hline
\end{tabular}

Signore et al.: Infliximab-Associated Chronic Inflammatory Central Nervous System Disease and Peroneal Nerve Injury in a Psoriatic Patient Refractory to Treatment

-13 Weisman MH, Moreland LW, Furst DE, Weinblatt ME, Keystone EC, Paulus HE, Teoh LS, Velagapudi RB, Noertersheuser PA, Granneman GR, Fischkoff SA, Chartash EK: Efficacy, pharmacokinetic and safety assessment of adalimumab, a fully tumor-necrosis factor-alpha monoclonal antibody, in adults with rheumatoid arthritis receiving concomitant methotrexate: a pilot study. Clin Ther 2003;25:1700-1721.

14 Segal BM, Cross AH: Fas(t) track to apoptosis: TNF receptors may suppress or potentiate CNS demyelination. Neurology 2000;55:906-907.

-15 Robinson WH, Genovese MC, Moreland LW: Demyelinating and neurologic events reported in association with tumor necrosis factor alpha antagonism: by what mechanisms could tumor necrosis factor alpha antagonists improve rheumatoid arthritis but exacerbate multiple sclerosis? Arthritis Rheum 2001;44:1977-1983.

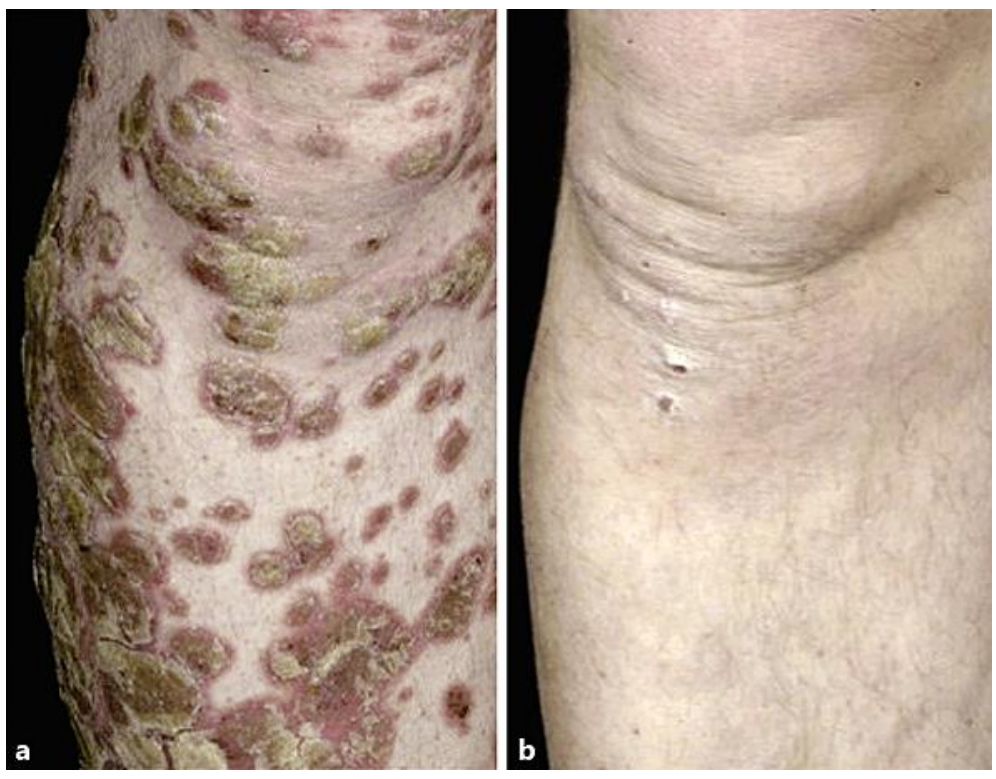

Fig. 1. a Sharply delineated erythematosquamous psoriatic plaques of the right leg before infliximab therapy in May 2008. b After the fourth infusion of infliximab at a dose of $5 \mathrm{mg} / \mathrm{kg}$ in February 2009, the psoriatic lesions on the right leg had almost disappeared. 


\section{Case Reports in Neurology}
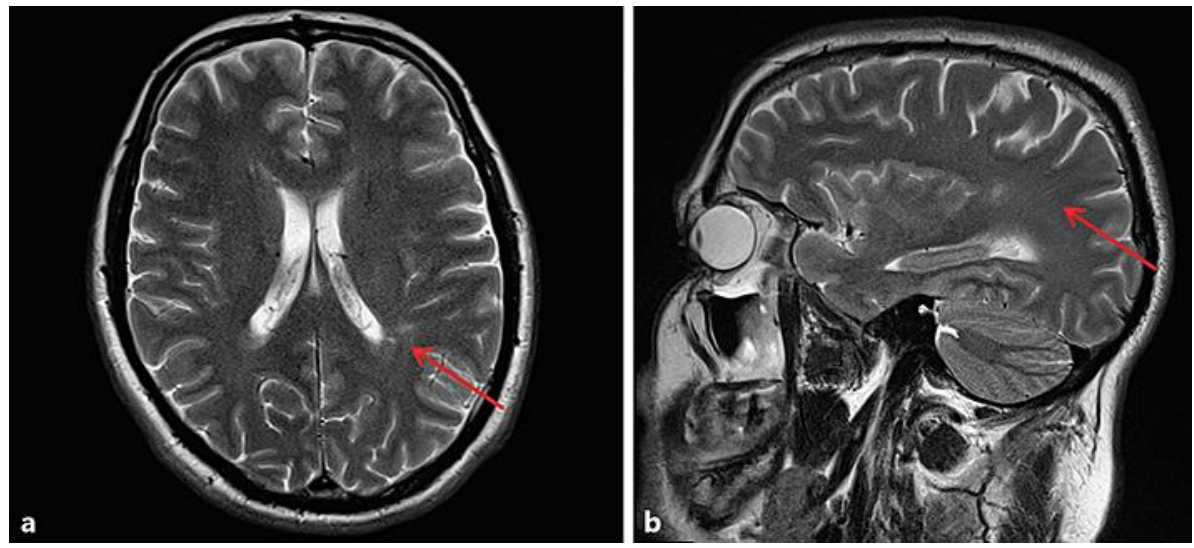

Fig. 2. a, b Magnetic resonance images of the brain in February 2009. T2-weighted images show a lesion in the left peritrigonal region without contrast agent enhancement (arrows) indicative of an inflammatory lesion. 